\title{
Impact of Transceiver Subsystems on Digital Back Propagation Performance
}

\author{
Lidia Galdino, Daniel Semrau and Polina Bayvel \\ Optical Networks Group, Department of Electronic and Electrical Engineering, \\ UCL (University College London), Torrington Place, London WC1E 7JE, UK. \\ Email: 1.galdino@ucl.ac.uk
}

\begin{abstract}
The potential, limitations and practicalities of digital back propagation is investigated in the presence of noise arising from amplifier spontaneous emission noise a well as from a nonideal transceiver subsystem.
\end{abstract}

\section{INTRODUCTION}

Nonlinear interference noise (NLI) induced by the Kerr effect in optical fibres is a significant factor limiting the amount of data that can be reliably transmitted through an conventional optical fibre communication system. For over an decade, many techniques and algorithms aiming to compensate optical fibre nonlinearity have been proposed and studied. The digital signal processing DSP-based optical fiber nonlinearity compensation (NLC) algorithm known as digital backpropagation (DBP) is one of the most popular NLC scheme and it is of particular interest. DBP extend the physical link with a virtual link in the DSP stage using an inverted propagation equation. It can be implemented on the transmitter-side, receiver-side or evenly split between them [1] [2] [3].

The fundamental performance limits of DBP have been extensively explored in several literature contributions. For several years, it was though that the main DBP performance bottlenecks were the presence of signal-noise NLI iteration which occur due to the amplified spontaneous emission (ASE) noise added by optical amplifiers along the transmission link, and stochastic effects, such as polarization mode dispersion (PMD). Only recently, it be shown that there is also a nonlinear interaction between the non-ideal transceiver subsystem, with a constrained-SNR (hereafter, transceiver noise) and the signal in the optical fibre transmission link [2] [3]. It has been identified as main reason for the discrepancy between theoretical prediction and experimental demonstration of fullfield FF-DBP (DBP is applied jointly to all received channels) performance. It has also been shown that transceiver-noise signal interference is the main noise source that limits the DBP performance at short or even at ultra-long-haul links [2].

In this paper, FF-DBP performance in the presence of transceiver noise is studied. The virtual transceiver-noise signal nonlinear interference is described, and a generalized expression to estimate the signal-to-noise ratio (SNR) obtained using FF-DBP in the presence of transceiver noise is described.

\section{TRANSCEIVER-NOISE SIGNAL NONLINEAR INTERFERENCE PHENOMENON}

Figure 1 illustrates the accumulation of nonlinear signalnoise interference along the transmission link. The transmitter

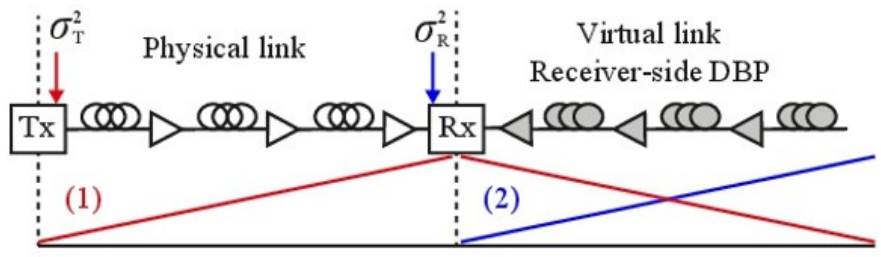

Fig. 1. Nonlinear signal-noise interactions accumulation along transmission link for receiver-side DBP. The red lines (1) show nonlinear transmitter noisesignal beating, cancelled after DBP, and the blue line (2) shows the nonlinear receiver noise-signal beating, generated after DBP

noise $\sigma_{\mathrm{T}}^{2}$ and receiver noise $\sigma_{\mathrm{R}}^{2}$ introduce an additional nonlinear noise-signal beating. If DBP is applied at the receiver side, as shown in Fig. 1, it compensates only for the nonlinear beating noise introduced by the transmitter (red line). This noise beats with the signal along the entire physical forward link and the beating is reversed in the virtual backward link during DBP. The transmitter noise-signal beating is completely canceled as the physical and virtual link exhibit the exact same distance. However, the noise that is introduced by the receiver (blue line) only beats with the signal along the virtual link. Therefore, only the receiver noise contributes to the beating term for receiver-side DBP.

In order to account for transceiver noise, a linear and a nonlinear interference term that accounts for the beating between the signal and transceiver noise must be taken into account. A generalized expression for the SNR after FF-DBP estimation is given by [2, Eq. (6)]

$$
\mathrm{SNR}_{\mathrm{FF}-\mathrm{DBP}}=\frac{P}{\kappa P+N P_{\mathrm{ASE}}+3 \eta\left(\kappa_{\mathrm{R}} \xi_{T R X} P+\xi_{A S E} P_{\mathrm{ASE}}\right) P^{2}} .
$$

where $P$ is the launch power per channel, $N$ is the number of spans, $P_{\mathrm{ASE}}$ is the ASE noise per amplifier, $\eta$ is the nonlinear interference coefficient for one span. The quantity $\xi_{T R X}$ is the transceiver noise beating accumulation factor, and $\xi_{A S E}$ is the ASE noise beating accumulation factor. $\kappa=\frac{1}{\mathrm{SNR}_{\mathrm{TRX}}}$, where $\mathrm{SNR}_{\mathrm{TRX}}$ is the maximum SNR that can be achieved in a given transceiver sub-system, and it can be measured in a back-to-back configuration. $\kappa_{R}$ is the ratio between the transmitter noise and receiver noise contribution, where $\kappa_{R}=0$ means that all the transceiver noise is generated by the transmitter (receiver-less noise) and $\kappa_{R}=1$, means that all the noise comes from the receiver. In other words, the transmitter noise contribution limits a maximum achievable 


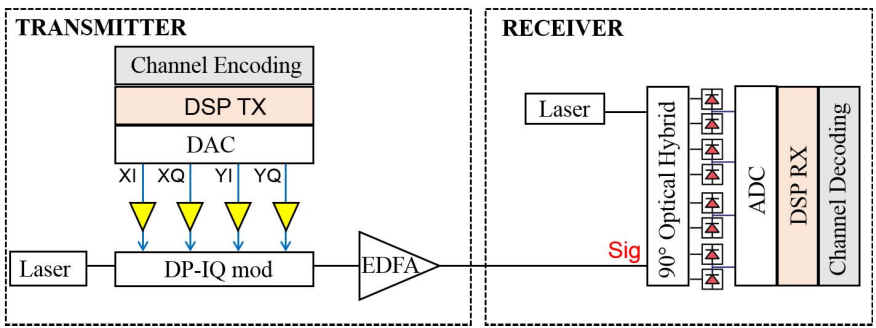

Fig. 2. Typical single-channel back-to-back implementation

$\mathrm{SNR}$ of $\mathrm{SNR}_{\mathrm{TX}}=\frac{\mathrm{SNR}_{\mathrm{TRX}}}{1-\kappa_{R}}$ and the receiver-side imposes a maximum achievable SNR of $\mathrm{SNR}_{\mathrm{RX}}=\frac{\mathrm{SNR}_{\mathrm{TRX}}}{\kappa_{R}}$.

\section{Abstraction of Transmitter And Receiver NOISE CONTRIBUTION}

In order to analysis different noise sources contribution in a transceiver sub-system, Fig. 2 illustrates a typical schematic of single channel back-to-back (transmitter connected straight to the receiver) sub-system. On the transmitter side, code modulation and digital signal processing (DSP) are applied off-line, for the multi-level drive mQAM signal generation. Digital preemphasis applied to the signal is also required to compensate for the frequency response of the transmitter components. The resulting in-phases(I) and quadrature $(\mathrm{Q})$ signals for each polarisation are them output using four digital-to-analog convertes (DACs) and subsequently amplified using four linear amplifiers, before being applied to a dualpolarisation (DP) IQ-modulator. The output of an laser is passed directly into the modulator before being optically amplified to form a DP-mQAM optical carrier.

On the receiver-side, the DP-mQAM signal are passed into the signal port of the digital coherent receiver, comprised by a $90^{\circ}$ optical hybrid, followed by balanced photodiodes, transimpedance amplifier (TIA) and a real-time digital sampling oscilloscope. DSP is applied off-line; initially, to compensated for receiver skew and different responsivities of the balance photodiodes, followed by standard DSP to recover the signal.

The transceiver noise contribution can be express as in eq. (2). where, the transmitter noise contribution $\left(\mathrm{SNR}_{\mathrm{TX}}\right)$, (eq. (3)) comes from non-ideal transmitter-side DSP, effective number of bits (ENOB) from digital to analog converters (DACs), noise figure from the linear amplifiers and ASE noise from the optical amplifier. On the receiver-side, the receiver noise source contribution (eq. 4) comes from ENOB from the analog to digital converters (ADCs), noise figure from the TIA amplifiers and non-ideal receiver-side DSP.

$$
\begin{gathered}
\frac{1}{\mathrm{SNR}_{\mathrm{TRX}}}=\frac{1}{\mathrm{SNR}_{\mathrm{TX}}}+\frac{1}{\mathrm{SNR}_{\mathrm{RX}}} \\
\frac{1}{\mathrm{SNR}_{\mathrm{TX}}}=\frac{1}{\mathrm{SNR}_{\mathrm{DSP}_{\mathrm{TX}}}}+\frac{1}{\mathrm{SNR}_{\mathrm{DAC}}}+\frac{1}{\mathrm{SNR}_{\mathrm{Amp}}}+\frac{1}{\mathrm{SNR}_{\mathrm{ASE}}} \\
\frac{1}{\mathrm{SNR}_{\mathrm{RX}}}=\frac{1}{\mathrm{SNR}_{\mathrm{ADC}}}+\frac{1}{\mathrm{SNR}_{\mathrm{TIA}}}+\frac{1}{\mathrm{SNR}_{\mathrm{DSP}_{\mathrm{RX}}}}
\end{gathered}
$$

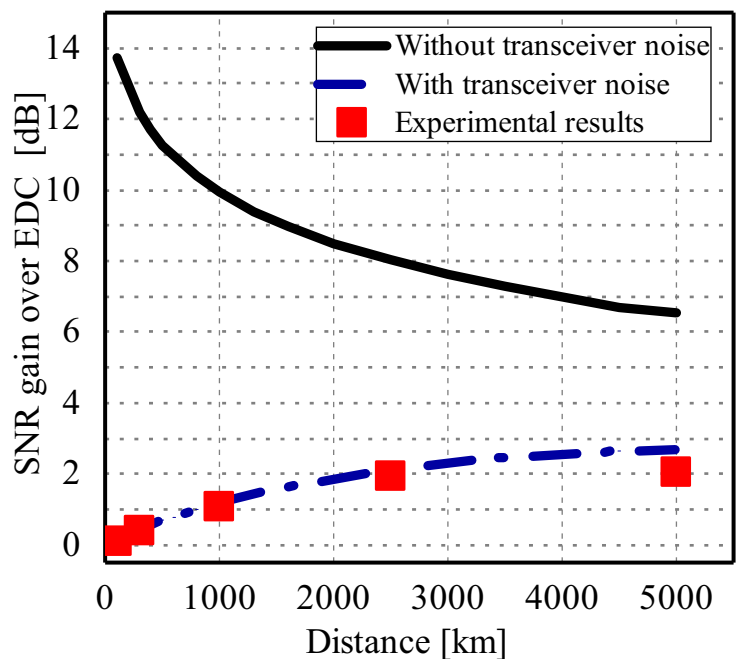

Fig. 3. SNR gain for applying FF-DBP over electronic chromatic dispersion (EDC) as a function of distance. Details of the transmission system under test can be found at [2].

Each noise source contribution from a transceiver subsystem can be identified, however, it is not straight forward to abstract exactly what is the transmitter and receiver noise contribution, as $\mathrm{SNR}_{\mathrm{TRX}}$, is the only directed measurement.

Fig. 3 illustrates the analytical prediction of SNR gain for FF-DBP (receiver side) with respect to electronic chromatic dispersion (EDC) compensation only. Details of transmission system under investigation can be found at [xxx]. It considered an transmission system without transceiver noise, and with transceiver noise. The experimental results are also displayed, showing a very good agreement with the analytical prediction using the recently proposed generalized expression to estimate the SNR for DBP in the presence of transceiver noise.

This simple expression enabled, for the first time, the prediction of the DBP performance for any practical system, which is not dominated by polarisation mode dispersion (PMD). Crucially, this model shows that the SNR gains from FFDBP can be significantly increased, provided the transceiver noise can be reduced. Finally, a new generalised expression that accurately predict the optimum NLC split between the transmitter and receiver on the presence of transceiver noise have been recently proposed in [3], where a completely analysis of the split NLC performance gain compared with transmitter-side and receiver-side DBP is reported.

\section{ACKNOWLEDGMENT}

This work was supported by the Royal Academy of Engineering under the Research Fellowships scheme The support from EPSRC INSIGHT and TRANSNET is acknowledged.

\section{REFERENCES}

[1] E. Temprana, et al. "Two-fold transmission reach enhancement enabled by transmitter-side digital backpropagation and optical frequency combderived information carriers," Optics Express23(16), 2015.

[2] L. Galdino, et al., "On the limits of digital back-propagation in the presence of transceiver noise", Opt. Express, 25(4), 2017.

[3] D. Semrau, et al., "The Impact of Transceiver Noise on Digital Nonlinearity Compensation", J. Lightw. Technol. 36(3), 2018. 